\title{
reviscafuences
}

ISSN: 1575-7072 | e-ISSN: 2172-7775

Páginas: $37-52$

Recibido: 2019-12-14

Revisado: 2019-11-06

Aceptado: 2020-01-16

Preprint: 2020-04-20

Publicación Final: 2020-06-15 www.revistascientificas.us.es/index.php/fuentes/index

DOI: https://doi.org/10.12795/revistafuentes.2020.v22.i1.04

\section{Mobile learning en las diferentes etapas educativas. Una revisión bibliométrica de la producción científica en Scopus (2007-2017)}

\author{
Mobile learning in the different educational stages. Bibliometric review of scientific \\ production in Scopus (2007-2017)
}

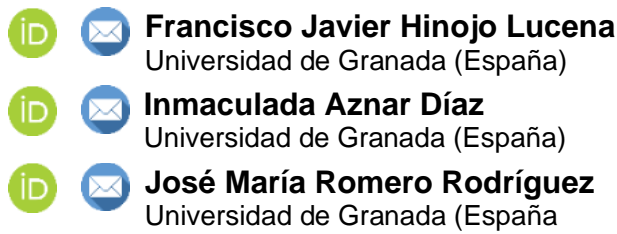

\begin{abstract}
Resumen
Los dispositivos móviles han irrumpido con fuerza en la sociedad y su aplicación en el ámbito educativo es una de sus consecuencias. La metodología mobile learning se posibilita como un recurso para la enseñanza-aprendizaje que puede traer consigo múltiples beneficios y en este estudio se ha realizado una revisión sobre la producción científica del mobile learning en las diferentes etapas educativas entre los años 2007 y 2017 . Para ello, se ha seguido una metodología propia de los estudios bibliométricos a partir del establecimiento de una serie de palabras clave para su consulta en la base de datos Scopus $(n=697)$. De modo que atendiendo a estudios previos se han analizado nueve variables relativas a: 1$)$ área científica; 2) número de publicaciones por año; 3) tipología del documento; 4) procedencia de los archivos; 5) palabras clave relacionadas; 6) instituciones; 7) países; 8) autores con más producción científica y 9) artículos más citados. Respecto a los resultados obtenidos, se ha determinado el creciente interés de la temática en los años 2015,2016 y 2017, donde se concentra la mayor parte de la literatura científica publicada. Sin embargo, predomina la producción en la etapa de Educación Superior sobre las demás. Finalmente, se constata que aunque los informes apuntan a la implementación del mobile learning en menos de un año, todavía queda camino por recorrer en la investigación enfocada a etapas educativas iniciales y medias.
\end{abstract}

\section{Abstract}

Mobile devices have burst into society and their application in education is one of the consequences. Mobile learning methodology is a teaching-learning resource that can bring multiple benefits. In this study, a review was conducted on the scientific production of mobile learning in the different educational stages between 2007 and 2017 . To this end, a methodology specific to bibliometric studies has been followed, based on the establishment of a series of key words for consultation in the Scopus database $(n=697)$. So, according to previous studies, nine variables have been analyzed in relation to: 1) scientific area; 2) number of publications per year; 3) typology of the document; 4) source of the archives; 5) related keywords; 6) institutions; 7) countries; 8) authors with more scientific production and 9) most cited articles. Regarding the results obtained, the growing interest of the subject in the years 2015, 2016 and 2017 has been determined, where most of the published scientific literature is concentrated. However, production in the Higher Education stage predominates over the others. Finally, it is noted that although reports point to the implementation of mobile learning in less than a year, there is still some way to go in research focused on initial and medium educational stages.

\section{Palabras clave / Keywords}

mobile learning, dispositivos móviles, sistema educativo, estudio bibliométrico, producción científica mobile learning, mobile devices, education system, bibliometric study, scientific production 


\section{Introducción}

El término mobile learning traducido al español como aprendizaje móvil, se define como el aprendizaje que se produce a través de la mediación de los dispositivos móviles (principalmente smartphones y tablets) (Aznar, Cáceres y Romero, 2018). En los últimos años esta metodología docente está teniendo gran protagonismo entre las Tecnologías de la Información y Comunicación (TIC) y la tendencia va en aumento, con la finalidad de instaurarse totalmente como un recurso metodológico más dentro del abanico docente. En esta línea, el informe Horizon (informe referente en materia de tecnología educativa) destaca en su última edición de 2017 las tecnologías emergentes que tendrán un mayor impacto en la educación en los próximos años (INTEF, 2017), resaltando al mobile learning como una metodología que se aplicará a corto plazo, concretamente en menos de un año (Adams et al., 2017).

El objetivo de este trabajo ha sido revisar la literatura científica sobre mobile learning en las distintas etapas educativas durante el periodo comprendido entre 2007 y 2017. La elección del periodo de inicio no es casual, a partir del año 2007 se inició el auge de los smartphones con el lanzamiento del iPhone y su sistema operativo iOS. Mientras que un año más tarde (2008) salía al mercado el sistema Android, por lo cual es oportuno iniciar la revisión de la bibliografía en el año en el que comenzó este fenómeno mundial. No obstante, el análisis realizado permite, entre otras variables, conocer la evolución de la producción científica por años dependiendo de la etapa educativa, con lo que arroja datos relevantes sobre su interés y posible implementación tanto en los niveles de Educación Infantil, Educación Primaria, Educación Secundaria y Educación Superior.

Ante este escenario y dada la tónica habitual de los informes Horizon (Johnson, Adams y Cummins, 2012; Johnson et al., 2013; 2014; 2015; 2016; Adams et al., 2017; 2018; Alexander et al., 2019) (tabla 1), se hace necesario un análisis de la producción científica para comprobar realmente la evolución que está teniendo el mobile learning.

Tabla 1

Predicciones sobre la implementación del mobile learning en los informes Horizon (2012-2019)

\begin{tabular}{ccc}
\hline Año del informe Horizon & Tecnología relacionada con el mobile learning & $\begin{array}{c}\text { Predicción de } \\
\text { implementación }\end{array}$ \\
\hline 2012 & Aplicaciones móviles y tablets & Un año o menos \\
2013 & Tablets & Un año o menos \\
2014 & Urae tu propio dispositivo (BYOD) & Uño o menos \\
2015 & Trae tu propio dispositivo (BYOD) & Uño o menos \\
2016 & Trae tu propio dispositivo (BYOD) & Un año o menos \\
2017 & Mobile Learning & Un año o menos \\
2018 & Mobile Learning & Un año o menos \\
2019 & Mobile Learning & \\
\hline
\end{tabular}

Por su parte, el concepto Bring Your Own Device (BYOD), que hace referencia al hecho de traer el propio dispositivo a clase en lugar de ser el centro quien los proporcione, es una de las principales estrategias para que sea efectiva la implementación del mobile learning dentro de las instituciones educativas (Romero, Trujillo y Rodríguez-García, 2018).

Por otro lado, esta metodología se define principalmente por estas cuatro características básicas que lo convierten en un potente recurso para favorecer el desarrollo del proceso de enseñanza-aprendizaje:

- Ubicuidad: capacidad del terminal, debido a sus funcionalidades y tamaño, para poder consultarse en cualquier momento y lugar.

- Trabajo cooperativo: facilidad para la cooperación entre estudiantes a través de aplicaciones de apoyo como Google Drive y Dropbox.

- Multiplicidad: opcionalidad que permite el dispositivo en función de la inmensa cantidad de recursos y aplicaciones móviles (apps) disponibles (Romero y Rodríguez-García, 2017).

- Autorregulación: libertad en la autonomía del estudiante para poder planificar cuando y donde realizar la tarea.

En suma, los dispositivos móviles son un elemento imprescindible en la sociedad y una tecnología emergente en el sistema educativo que poco a poco se está abriendo paso y por ello, debe ser objeto de análisis con el fin de integrarse como un elemento más del currículo (Jiménez, Mora y Cuadros, 2016; González y Gutiérrez, 
2017). En este escenario, el gobierno de la Junta de Andalucía ha incentivado la dotación de herramientas digitales (ordenadores portátiles y aulas digitales) en la Educación Primaria y Educación Secundaria durante los años 2009 y 2011 a través del programa escuela TIC 2.0 (Consejería de Educación de la Junta de Andalucía, 2011). A su vez, el Ministerio de Educación, Cultura y Deporte del Gobierno de España estableció en el curso académico 2009/2010 que los centros debían promover la integración de las TIC. Para este fin, se generó el programa Escuela 2.0 que se encargó de dotar de recursos tecnológicos a los centros escolares de primaria y secundaria y formar a los docentes (Ministerio de Educación, Cultura y Deporte (Ministerio de Educación, Cultura y Deporte, 2009).

\section{Metodología}

Este trabajo de investigación sigue una metodología propia de los estudios bibliométricos (Glanzel y Schoepflin, 1999). Esto es, con carácter descriptivo y basada en la descripción de una serie de variables proporcionadas por la base de datos Scopus, que posibilitan datos cuantitativos sobre el estado de la cuestión acerca de un determinado tema (Urrútia y Bonfill, 2010; López, Vázquez y Román, 2015). En este caso, sobre la producción científica del mobile learning en las diferentes etapas educativas. Para ello, se establecieron cinco palabras clave indexadas en el Tesauro ERIC y que responden al objetivo de investigación, éstas son: Mobile Learning, Preschool Education, Primary Education, Secondary Education y Higher Education.

Cabe resaltar que la elección de la base de datos Scopus corresponde a su capacidad aglutinadora, siendo la mayor base de datos de resúmenes (más de 28 millones). De tal forma que representa el $80 \%$ de las publicaciones internacionales revisadas por expertos y con índice de impacto (Scientific Journal Rankings SJR). Por tanto, los resultados obtenidos nos muestran una panorámica general del mobile learning a nivel mundial, a partir de la producción científica de impacto.

\subsection{Muestra}

La unidad de análisis se compuso por toda la literatura científica indexada en Scopus desde 2007 a 2017 sobre mobile learning en las distintas etapas educativas: Educación Infantil, Educación Primaria, Educación Secundaria y Educación Superior. Para ello, se aplicó como principal criterio de inclusión los años de publicación (2007-2017). Por tanto, el criterio de exclusión fue que la literatura no estuviera publicada entre ese periodo temporal. El diagrama de flujo muestra el proceso de refinado (figura 1).



Figura 1. Diagrama de flujo

Nota: $\mathbf{n}=$ muestra $\left(\mathrm{n}^{\mathbf{0}}\right.$ de resultados) 
La introducción de la ecuación de búsqueda en el motor de búsqueda de Scopus, muestra un panorama bastante heterogéneo, donde la gran mayoría de producción científica se concentra en la etapa de Educación Superior (tabla 2).

\section{Tabla 2}

Número de resultados con filtros del descriptor mobile learning con cada etapa educativa

\begin{tabular}{|c|c|c|}
\hline Ecuación de búsqueda & $\mathbf{n}$ & $\%$ \\
\hline "Mobile Learning" OR M-learning AND "Preschool Education" & 4 & .57 \\
\hline “Mobile Learning" OR M-learning AND "Primary Education” & 22 & 3,15 \\
\hline "Mobile Learning” OR M-learning AND “Secondary Education” & 52 & 7,46 \\
\hline "Mobile Learning" OR M-learning AND "Higher Education" & 619 & 88,8 \\
\hline
\end{tabular}

\subsection{Análisis de datos}

El análisis de datos se realizó en función de la búsqueda realizada a partir de las cuatro ecuaciones de búsqueda. Asimismo, "Mobile Learning" se unificó a través del operador booleano "AND" con el resto de palabras clave que responden a las etapas del sistema educativo. De este modo, se determinó el estado de la literatura científica, desde 2007 hasta el año 2017, sobre el aprendizaje que se produce a través de los dispositivos móviles en los distintos niveles educativos. La recogida de datos tuvo lugar en el segundo semestre de 2018 por lo que se engloban los últimos 10 años de producción científica con 2017 inclusive.

En relación al descriptor "Mobile Learning" es utilizado en la revisión llevada a cabo sobre la producción científica del mobile learning en España durante el periodo de 2009-2013 realizada por Brazuelo y Gallego (2014). Del mismo modo que se vuelve a utilizar "Mobile Learning" y en este caso "Higher Education" para un estudio de caso sobre desarrollo de habilidades cognitivas con aprendizaje móvil en Educación Superior (Ramos, Herrera y Ramírez, 2010), publicado en la revista Comunicar, posicionada en el primer cuartil del Journal Citation Reports (JCR). Aunando en el panorama internacional, en la prestigiosa revista Computers and Education, posicionada en el primer cuartil (JCR), son varios los artículos (O'Bannon y Thomas, 2014; Zydney y Warner, 2015; Han, \& Shin, 2016) que recogen como palabra clave "Mobile Learning". En esta línea, autores relevantes debido al número de citas de sus artículos como Mike Sharples emplean el descriptor "Mobile Learning" en algunos de sus artículos (Sharples, Corlett y Westmancott, 2002; Corlett, Sharples, Bull y Chan, 2005). Por tanto, aunque el establecimiento de las palabras clave en primera instancia responden al tópico y objetivos del trabajo, se constata que "Mobile Learning" es un descriptor utilizado por autores y revistas de impacto, al igual que su adecuación para combinarlo con los descriptores referentes a los niveles educativos.

Por su parte, respecto a las variables de análisis, éstas se han seleccionado en base a los estudios previos de Rodríguez-García y Martínez (2017) y Mengual, Vázquez y López (2017). En total se recogieron nueve:

1. Área científica.

2. Número de publicaciones por año.

3. Tipología del documento.

4. Procedencia de los archivos.

5. Palabras clave relacionadas.

6. Instituciones.

7. Países.

8. Autores con más producción científica.

9. Referencias más citadas.

\section{Resultados}

En relación con la variable 1. Área científica, de donde proviene la literatura. Predominan las Ciencias Computacionales (CC. Computacionales) en Educación Infantil (60\%), de igual forma siguen prevaleciendo en Educación Primaria (46\%) (figura 2). En Educación Secundaria sigue manteniéndose como principal el área de Ciencias Computacionales (44\%) pero la diferencia con las Ciencias Sociales (CC. Sociales) es escasa $(41 \%)$. Por último, en Educación Superior se repite la misma estela anterior, Ciencias Computacionales (43\%) y Ciencias Sociales (39\%) (figura 3). 
Educación Infantil

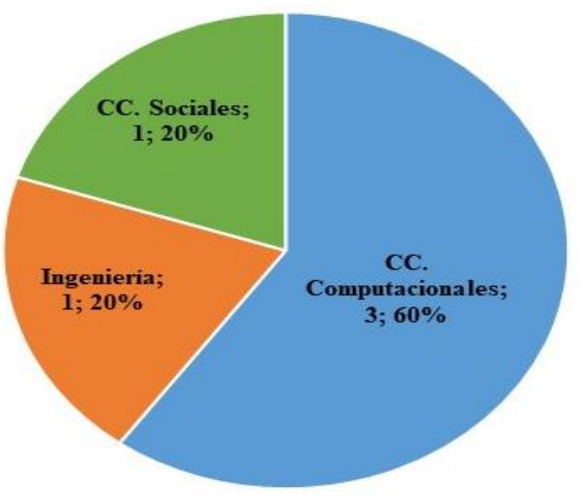

Educación Primaria



Figura 2. Principales áreas científicas de prevalencia en Educación Infantil y Educación Primaria

Educación Secundaria



Educación Superior

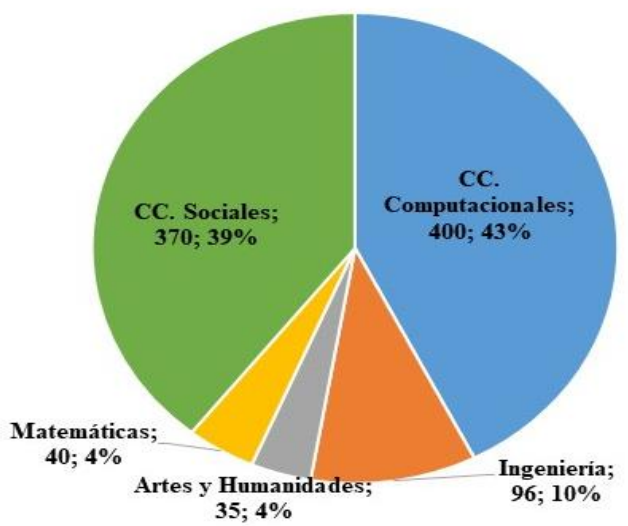

Figura 3. Principales áreas científicas de prevalencia en Educación Secundaria y Educación Superior

Respecto a la variable 2. Número de publicaciones por año, la mayoría de publicaciones de mobile learning tanto en Educación Infantil, Educación Primaria y Educación Superior se sitúan entre los años 2015-2017. En referencia a la ley de crecimiento exponencial de Price (1986), la cual indica que la producción tiende a duplicarse cada 10 años, la producción científica en la etapa de Educación Infantil se encuentra en la etapa de precursores, siendo esta la fase inicial en la que comienza la producción, de modo ha iniciado en el año 2015 con una publicación (25\%) y su producción máxima se sitúa en el año 2016 con dos referencias (50\%) (figura 4).

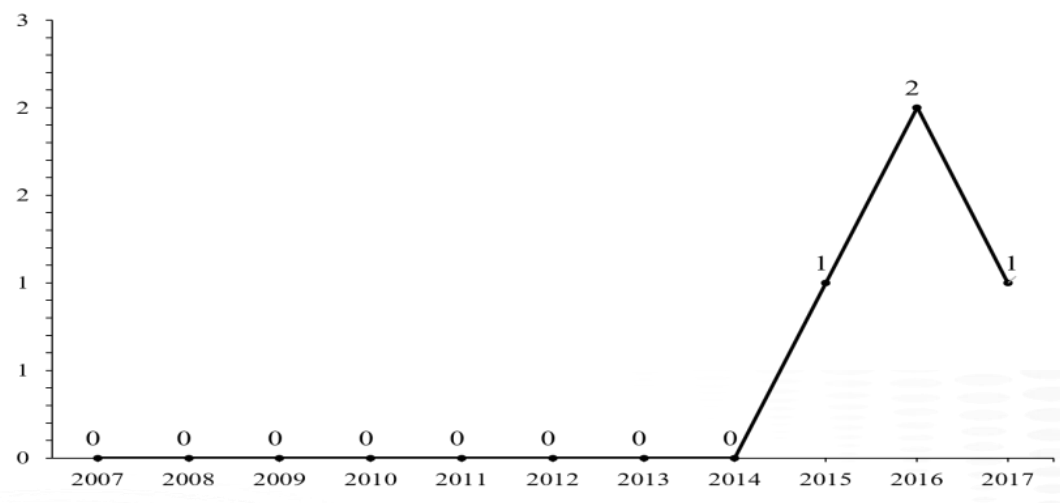


Figura 4. Número de publicaciones por año sobre mobile learning en Educación Infantil

En cambio, en Educación Primaria los inicios se sitúan en 2010 con 4 documentos (18,18\%). Su producción máxima fue en 2016 con cinco publicaciones (22,7\%) y mínima en 2012 con una referencia (4,5\%). Debido al bajo número de documentos y de acuerdo a Price (1986) la producción se encontraría, al igual que con Educación Infantil, en una fase de precursores (figura 5).



Figura 5. Número de publicaciones por año sobre mobile learning en Educación Primaria

En Educación Secundaria, el mínimo de producción lo encontramos en el año de inicio 2008 y en 2010 con dos referencias $(3,84 \%)$ y el máximo en el año 2014 con 13 documentos $(25 \%)$. En cuanto a la ley Price (1986), encontramos igual que en los casos anteriores que la producción no termina de despegar, situándose en una fase inicial (figura 6).

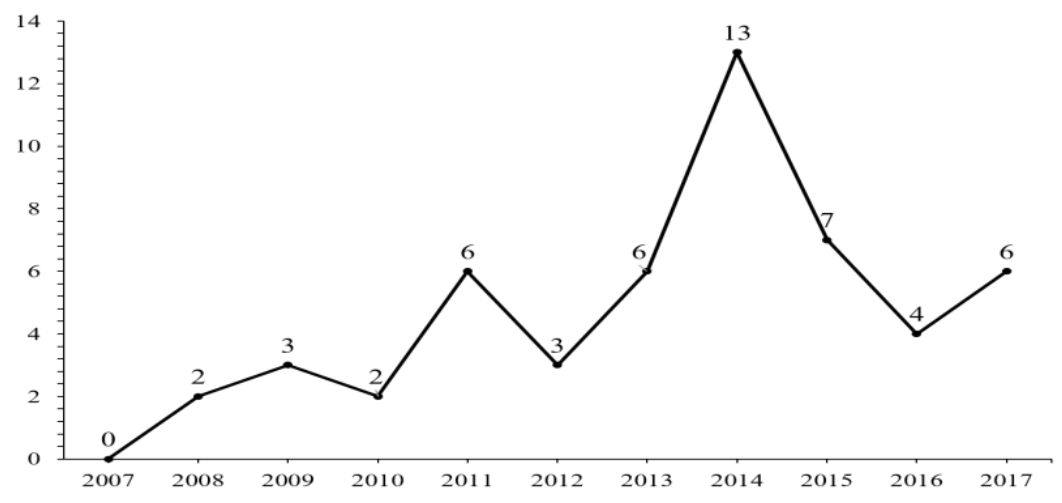

Figura 6. Número de publicaciones por año sobre mobile learning en Educación Secundaria

Por último, la etapa de Educación Superior es la que tiene más desarrollo observándose las tres etapas en la producción científica (Price, 1986): etapa de precursores (2007-2009), etapa de crecimiento exponencial (2010-2015) y etapa de crecimiento lineal (2016-2017). Asimismo, como destaca la ley de Price (1986) a los 10 años se duplica la producción de la literatura como podemos observar en este caso (2007 - 11 documentos y 2017 - 91 documentos). En relación a la producción máxima esta se sitúa en el año 2015 con 120 referencias $(19,4 \%)$ y la mínima en 2007 con 11 (1,77\%), la media de publicaciones por año es de 61,8 (figura 7 ). 


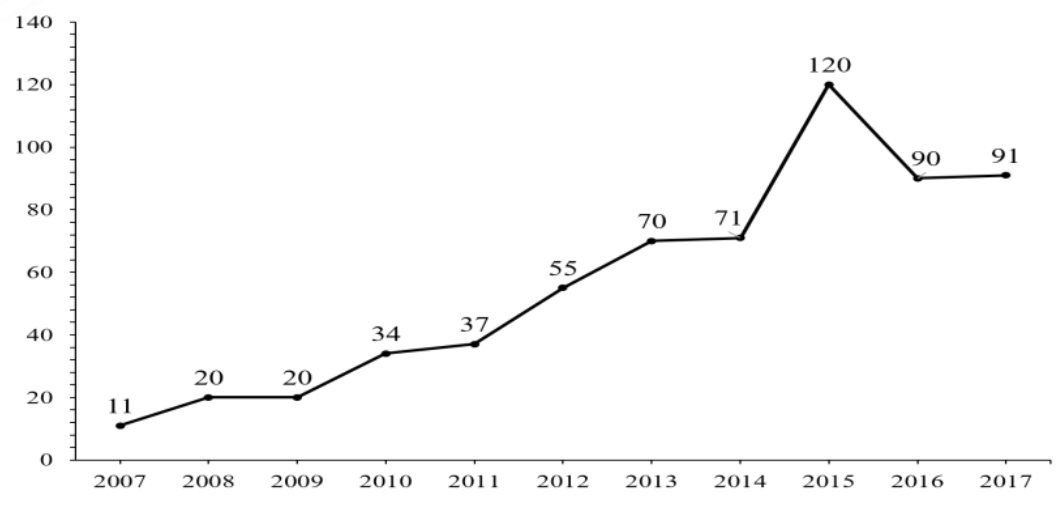

Figura 7. Número de publicaciones por año sobre mobile learning en Educación Superior

Por su parte, en la variable 3. Tipología del documento, prevalece en la producción científica de Educación Infantil los artículos (50\%) y en Educación Primaria las aportaciones a conferencias (68\%) (figura 8). En cambio, en Educación Secundaria predominan los artículos (52\%) sobre las aportaciones a conferencias (33\%) y en Educación Superior se invierte superando las contribuciones a conferencias (43\%) a los artículos de revista (40\%) (figura 9).
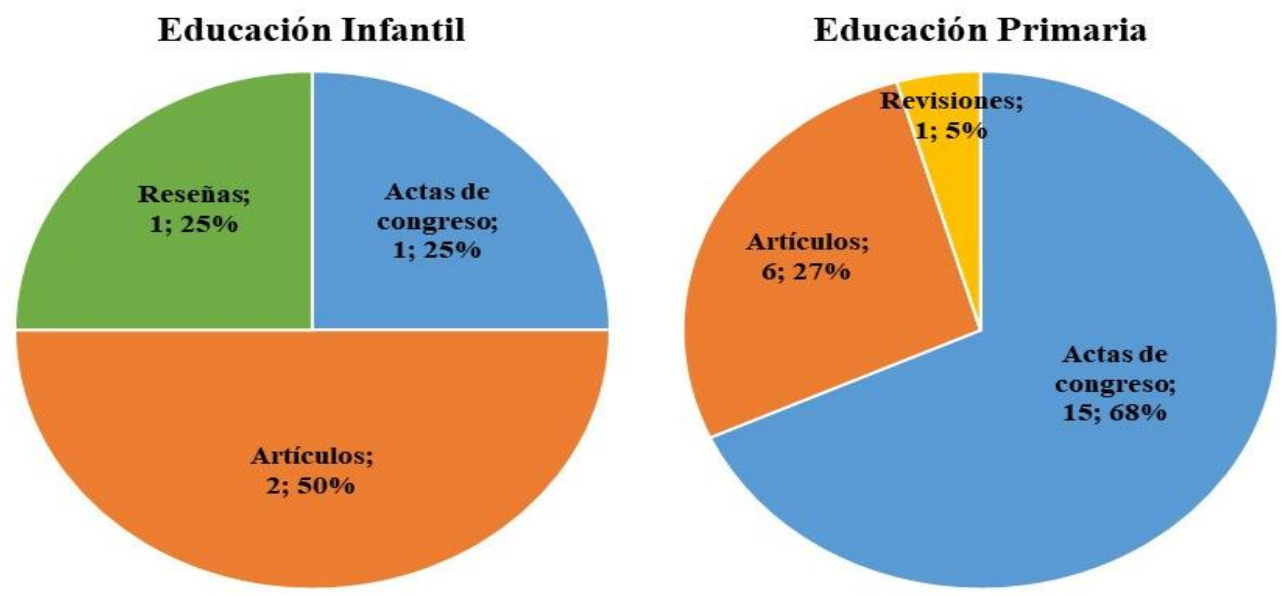

Figura 8. Tipología de la producción científica en Educación Infantil y Educación Primaria

\section{Educación Secundaria}



Educación Superior

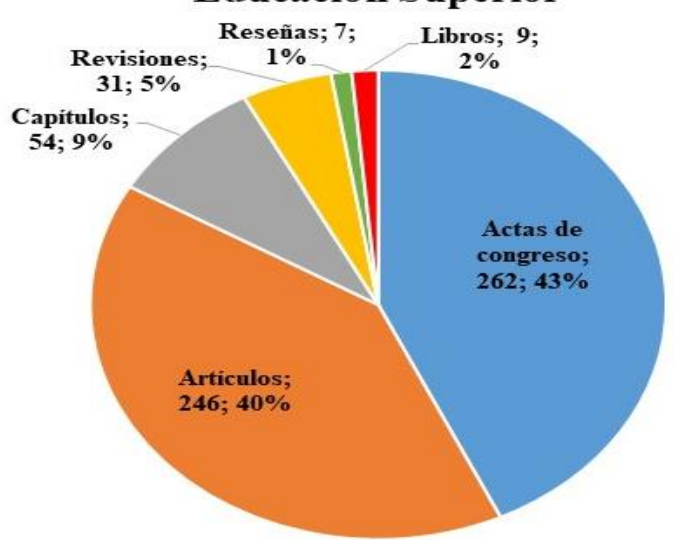

Figura 9. Principales tipologías de la producción científica en Educación Secundaria y Educación Superior 
En cuanto a la variable 4. Procedencia de los archivos, en la etapa de Educación Infantil el $50 \%$ de las publicaciones se concentran en dos artículos en International Journal Of Interactive Mobile Technologies, que a su vez cuenta con tres citas y un índice de impacto de 1,5. Por otro lado, en Educación Primaria el 13,63\% queda recogido en tres aportaciones a ACM International Conference, con 11 citas y un índice de impacto de 3,66 . La demás producción se diversifica en distintas conferencias y revistas, que no alcanzan a tener dos referencias. En la etapa de Educación Secundaria destaca la revista Computers and Education con 12 documentos que representan el $23 \%$, esta revista obtiene un alto índice de impacto de sus documentos (40) con 481 citas en total. En Educación Superior la fuente con más documentos es Communications In Computer And Information Science con 22 (3,55\%), 19 citas y un índice de impacto de 0,86, aunque destaca sobre las demás Computers and Education que con tan solo 12 documentos (1,94\%) obtiene 1.199 citas con un índice de impacto de 99,91 (tabla 3).

\section{Tabla 3}

Procedencia de la literatura científica con mayor número de referencias en las etapas de Educación Secundaria y Educación Superior

\begin{tabular}{|c|c|c|c|c|c|}
\hline Etapa & Título & $\mathbf{n}$ & $\%$ & $\mathbf{C}^{*}$ & $I^{*}$ \\
\hline Educación & Computers and Education & 12 & 23 & 481 & 40 \\
\hline \multirow[t]{3}{*}{ Secundaria } & $\begin{array}{l}\text { Proceedings Of The European } \\
\text { Conference On Games Based Learning }\end{array}$ & 4 & 7,69 & 5 & 1,25 \\
\hline & $\begin{array}{l}\text { ACM International Conference Proceeding } \\
\text { Series }\end{array}$ & 3 & 5,76 & 7 & 2,33 \\
\hline & $\begin{array}{l}\text { Proceedings Of The 10th International } \\
\text { Conference On Mobile Learning } 2014\end{array}$ & 3 & 5,76 & - & - \\
\hline \multirow[t]{5}{*}{$\begin{array}{l}\text { Educación } \\
\text { Superior }\end{array}$} & $\begin{array}{l}\text { Communications In Computer And } \\
\text { Information Science }\end{array}$ & 22 & 3,55 & 19 & .86 \\
\hline & Lecture Notes In Computer Science & 18 & 3,55 & 19 & 1,05 \\
\hline & $\begin{array}{l}\text { Handbook Of Mobile Teaching And } \\
\text { Learning }\end{array}$ & 16 & 2,58 & 11 & .68 \\
\hline & $\begin{array}{l}\text { International Journal Of Mobile Learning } \\
\text { And Organisation }\end{array}$ & 16 & 2,58 & 60 & 3,75 \\
\hline & Computers and Education & 12 & 1,94 & 1.199 & 99,91 \\
\hline
\end{tabular}

Nota: se han considerado únicamente aquellos títulos que tuvieran más de 2 referencias y/o los 5 más relevantes. $C^{*}=$ citas, $I^{*}=$ índice de impacto. Cálculo del índice de impacto = citas $/$ documentos.

Siguiendo con el análisis, en la variable 5. Palabras clave relacionadas, destaca en Educación Infantil como las más utilizadas: mobile learning (4 veces - 100\%) y preschool education (4 veces - 100\%). En Educación Primaria las más empleadas son mobile learning (12 veces - 54,5\%), Education (12 veces $-54,5 \%$ ) y teaching (12 veces - 54,5\%) (tabla 4). En Educación Secundaria como primera sigue situándose mobile learning (29 veces $-55,76 \%$ ), segunda teaching (23 veces - 44,2\%), mientras que en Educación Superior mobile learning (326 veces $-52,66 \%$ ) se mantiene como la más utilizada, seguida de higher education (272 veces - 43,9\%) (tabla 5). 
Tabla 4

Palabras clave más utilizadas en la producción científica en las etapas de Educación Infantil y Educación Primaria

\begin{tabular}{lllc}
\hline Etapa & Palabras clave & $\mathbf{n}$ & \% \\
\hline Educación Infantil & Mobile learning & 4 & 100 \\
& Preschool Education & 4 & 100 \\
& E-learning & 2 & 50 \\
& Education & 2 & 50 \\
& Engineering Education & 2 & 50 \\
\hline Educación Primaria & Mobile learning & 12 & 54,5 \\
& Education & 12 & 54,5 \\
& Teaching & 8 & 54,5 \\
& Primary Education & 8 & 36,3 \\
\hline
\end{tabular}

Nota: solo se han considerado las 5 primeras palabras clave.

Tabla 5

Palabras clave más utilizadas en la producción científica en las etapas de Educación Secundaria y Educación Superior

\begin{tabular}{llcc}
\hline Etapa & Palabras clave & $\mathbf{n}$ & \% \\
\hline Educación Secundaria & Mobile Learning & 29 & 55,76 \\
& Teaching & 23 & 44,2 \\
& E-learning & 22 & 42,3 \\
& Secondary Education & 21 & 40,3 \\
& Students & 21 & 40,3 \\
\hline Educación Superior & Mobile Learning & 326 & 272 \\
& Higher Education & 242 & 52,66 \\
& E-learning & 215 & 33,9 \\
& Education & 214 & 34,73 \\
\hline
\end{tabular}

Nota: solo se han considerado las 5 primeras palabras clave.

En la variable 6. Instituciones, que más literatura científica producen acerca del mobile learning en las distintas etapas educativas. Se sitúa referente a la etapa de Educación Infantil, el Demokritos National Centre for Scientific Research que tiene un total de tres referencias, correspondiendo al $75 \%$ de las publicaciones de mobile learning en dicha etapa. En Educación Primaria, la Universidad de Salamanca encabeza la lista de literatura científica con seis referencias, un $27,2 \%$ del total, que suman 11 citas con un índice de impacto de 1,83 (tabla 6). Asimismo, en Educación Secundaria el National Taiwan University of Science and Technology se sitúa como la más productora con cuatro referencias que representan un $7,69 \%$ de las publicaciones, entre todas ellas suman un total de 123 citas con un índice de impacto de 30,75. Finalmente, en la etapa de Educación Superior lidera la tabla la University of Southern Queensland de Australia con 14 referencias (2,26\%), que presenta 47 citas con un índice de impacto de 3,35 (tabla 7).

\section{Tabla 6}

Instituciones con más producción científica en la etapa de Educación Infantil y Educación Primaria

\begin{tabular}{|c|c|c|c|c|c|}
\hline \multirow{3}{*}{$\begin{array}{l}\text { Etapa } \\
\text { Educación } \\
\text { Infantil }\end{array}$} & Institución & $\mathbf{n}$ & $\%$ & $\mathbf{C}^{*}$ & $I^{*}$ \\
\hline & $\begin{array}{l}\text { Demokritos National Centre for Scientific } \\
\text { Research (Grecia) }\end{array}$ & 3 & 75 & 3 & 1 \\
\hline & $\begin{array}{l}\text { Institute of Informatics and } \\
\text { Telecommunications, Athens (Grecia) }\end{array}$ & 2 & 50 & 3 & 1,5 \\
\hline Educación & Universidad de Salamanca (España) & 6 & 27,2 & 11 & 1,83 \\
\hline Primaria & Universidad Politécnica de Cataluña (España) & 2 & 9 & - & - \\
\hline
\end{tabular}

Nota: se han considerado únicamente aquellas instituciones que tuvieran más de 2 referencias y/o los 5 más relevantes. $C^{*}=$ citas, $I^{*}=$ índice de impacto. Cálculo del índice de impacto $=$ citas $/$ documentos. 
Tabla 7

Instituciones con más producción científica en la etapa de Educación Secundaria y Educación Superior

\begin{tabular}{|c|c|c|c|c|c|}
\hline Etapa & Institución & $\mathbf{n}$ & $\%$ & $\mathbf{C}^{\star}$ & $I^{\star}$ \\
\hline \multirow[t]{5}{*}{$\begin{array}{l}\text { Educación } \\
\text { Secundaria }\end{array}$} & $\begin{array}{l}\text { National Taiwan University of Science and } \\
\text { Technology }\end{array}$ & 4 & 7,69 & 123 & 30,75 \\
\hline & Universdiad Pompeu Fabra (España) & 4 & 7,69 & 35 & 8,75 \\
\hline & Technology University of Graz (Austria) & 3 & 5,76 & 5 & 1,66 \\
\hline & National Taiwan Normal University & 3 & 5,76 & 81 & 27 \\
\hline & University of Amsterdam (Países Bajos) & 2 & 3,84 & 5 & 2,5 \\
\hline \multirow[t]{5}{*}{$\begin{array}{l}\text { Educación } \\
\text { Superior }\end{array}$} & $\begin{array}{l}\text { University of Southern Queensland } \\
\text { (Australia) }\end{array}$ & 14 & 2,26 & 47 & 3,35 \\
\hline & Universidad de Salamanca (España) & 13 & 2,1 & 39 & 3 \\
\hline & University of Aveiro (Portugal) & 10 & 1,61 & 7 & .7 \\
\hline & University of Cape Town (Sudafrica) & 9 & 1,45 & 24 & 2,66 \\
\hline & $\begin{array}{l}\text { University of Hradec Kralove (República } \\
\text { Checa) }\end{array}$ & 9 & 1,45 & 11 & 1,22 \\
\hline
\end{tabular}

Nota: se han considerado únicamente aquellas instituciones que tuvieran más de 2 referencias y/o los 5 más relevantes. $C^{*}=$ citas, $I^{*}=$ índice de impacto. Cálculo del índice de impacto = citas/documentos.

En relación con la variable 7 , "Países con mayor producción científica", en cada etapa varían los países con más documentos. Así pues, en Educación Infantil el único país con más de dos referencias es Grecia, que presenta tres documentos (75\%), con tres citas y un índice de impacto de 1 punto. Mientras que en Educación Primaria el país con más referencias es España (11 documentos - 50\%), que obtiene 76 citas de sus documentos (tabla 8). En Educación Secundaria sigue siendo el principal España con 11 documentos $(21,1 \%)$, 44 citas y un índice de impacto de 4 puntos, aunque Estados Unidos con cuatro documentos presenta un mayor índice de impacto (35.25). Por último, en Educación Superior el país con mayor producción científica es Reino Unido con 77 referencias (12,43\%) que obtienen 852 citas (índice de impacto de 11 puntos), sin embargo Estados Unidos con 69 documentos tiene mayor rendimiento (1.404 citas e índice de impacto de 20,34) (tabla 9).

Tabla 8

Países con más producción científica en la etapa de Educación Infantil y Educación Primaria

\begin{tabular}{llcccc}
\hline Etapa & País & $\mathbf{n}$ & $\%$ & $\mathbf{C}^{*}$ & $\mathbf{I}^{\boldsymbol{*}}$ \\
\hline Educación Infantil & Grecia & 3 & 75 & 3 & 1 \\
\hline Educación Primaria & España & 11 & 50 & 76 & 6,9 \\
& Grecia & 4 & 18,1 & 9 & 2,25 \\
\hline
\end{tabular}

Nota: se han considerado únicamente aquellos países que tuvieran más de 2 referencias y/o los 5 más relevantes. $C^{*}=$ citas, $I^{*}=$ índice de impacto. Cálculo del índice de impacto = citas $/$ documentos.

\section{Tabla 9}

Países con más producción científica en la etapa de Educación Secundaria y Educación Superior

\begin{tabular}{llcccc}
\hline Etapa & País & $\mathbf{n}$ & $\%$ & $\mathbf{C}^{\boldsymbol{*}}$ & $\mathbf{I}^{\boldsymbol{*}}$ \\
\hline Educación Secundaria & España & 11 & 21,1 & 44 & 4 \\
& Taiwán & 8 & 15,38 & 167 & 20,87 \\
& Austria & 5 & 9,61 & 9 & 1,8 \\
& Reino Unido & 5 & 9,61 & 166 & 33,2 \\
& Estados Unidos & 4 & 7,69 & 141 & 35,25 \\
\hline Educación Superior & Reino Unido & 77 & 12,43 & 852 & 11 \\
& Estados Unidos & 69 & 11,14 & 1.404 & 20,34 \\
& Australia & 57 & 9,2 & 254 & 4,45 \\
& España & 48 & 7,75 & 160 & 3,33 \\
& Malasia & 34 & 5,49 & 38 & 1,11 \\
\hline
\end{tabular}

Nota: se han considerado únicamente aquellos países que tuvieran más de 2 referencias y/o los 5 más relevantes. $C^{*}=$ citas, $I^{*}=$ índice de impacto. Cálculo del índice de impacto = citas $/$ documentos. 
En referencia a la variable 8. Autores con más producción científica, en Educación Infantil destacan dos autores (Drigas, A.S y Kokkalia, G) con un total de tres referencias (75\%) que suman un total de tres citas en sus documentos. En cambio, en Educación Primaria resalta como autores con más referencias SánchezPrieto, J.C y García-Peñalvo, F.J con un total de seis documentos (27,2\%), 20 citas y un índice de impacto de 3.3 (tabla 10). Respecto a la etapa de Educación Secundaria, los autores Hernández-Leo, D, Hwang, G.J y Melero, J presentan cuatro contribuciones cada uno (7.69\%), destacando entre ellos Hwang, G.J con 123 citas y un mayor índice de impacto (30.75). En Educación Superior, resaltan Farley, $\mathrm{H}$ y Murphy, A con 11 documentos (1,7\%), 32 citas y un índice de impacto de 2,9. Aunque Cochrane, T con ocho documentos $(1,29 \%)$ alcanza mayor número de citas (55) y por tanto, mayor índice de impacto (6.87) (tabla 11).

Tabla 10

Autores con más producción científica en la etapa de Educación Infantil y Educación Primaria

\begin{tabular}{llllll}
\hline Etapa & Autor & $\mathbf{n}$ & $\%$ & $\mathbf{C}^{*}$ & $\mathbf{I}^{\boldsymbol{*}}$ \\
\hline Educación Infantil & Drigas, A.S & 3 & 75 & 3 & 1 \\
& Kokkalia, G & 3 & 75 & 3 & 1 \\
\hline Educación Primaria & Sánchez-Prieto, J.C & 6 & 27,2 & 20 & 3,3 \\
& García-Peñalvo, F.J & 6 & 27,2 & 20 & 3,3 \\
& Migueláñez, S.O & 5 & 22,7 & 13 & 2,6 \\
\hline
\end{tabular}

Nota: se han considerado únicamente aquellos autores con más de 2 referencias y/o los 5 más relevantes. $C^{*}=$ citas, $I^{*}=$ índice de impacto. Cálculo del índice de impacto $=$ citas $/$ documentos .

\section{Tabla 11}

Autores con más producción científica en la etapa de Educación Secundaria y Educación Superior

\begin{tabular}{llllll}
\hline Etapa & Autor & $\mathbf{n}$ & $\%$ & $\mathbf{C}^{\boldsymbol{*}}$ & $\mathbf{I}^{\boldsymbol{*}}$ \\
\hline Educación Secundaria & Hernández-Leo, D & 4 & 7,69 & 35 & 8,75 \\
& Hwang, G.J & 4 & 7,69 & 123 & 30,75 \\
& Melero, J & 4 & 7,69 & 35 & 8,75 \\
& Blat, J & 3 & 5,76 & 24 & 8 \\
& Ebner, M & 3 & 5,6 & 5 & 1,6 \\
\hline Educación Superior & Farley, H & 11 & 1,7 & 32 & 2,9 \\
& Murphy, A & 11 & 1,7 & 32 & 2,9 \\
& Cochrane, T & 8 & 1,29 & 55 & 6,87 \\
& Ngambi, D & 8 & 1,29 & 24 & 3 \\
\hline
\end{tabular}

Nota: se han considerado únicamente aquellos autores con más de 2 referencias y/o los 5 más relevantes. $C^{*}=$ citas, $I^{*}=$ índice de impacto. Cálculo del índice de impacto = citas $/$ documentos .

Por último, en los datos de la variable 9, "Referencias más citadas", se recogen las referencias con más impacto, se ha tenido en cuenta que presentaran diez o más citas, excepto en Educación Infantil que la única referencia citada tiene tres citas. Así pues, se muestra la información sobre los autores, año, título de la aportación, medio de publicación y las citas recibidas. En Educación Infantil, el trabajo más citado es de 2016 y tiene como título "Mobile learning for special preschool education", el cual se cita un total de tres veces. Por otro lado, en Educación Primaria el artículo de 2013 "Evaluation of learning outcomes using an educational iPhone game vs. traditional game" es el que presenta mayor número de citas (54) (tabla 12). En Educación Secundaria resalta la contribución "iTunes University and the classroom: Can podcasts replace Professors?" de 2009 con 134 citas (tabla 13). Además, todos los artículos con un mayor número de citas provienen de la revista Computers and Education, sumando un total de 386 citas con un índice de impacto de 77,2. No obstante, cierra el análisis de la última variable la etapa de Educación Superior con 408 citas que obtiene el artículo titulado "Mobile learning: A framework and evaluation" del año 2007 (tabla 14). En suma, fijándonos en la literatura científica con más impacto en todas las etapas, la revista Computers and Education es la que alcanza un mayor índice de impacto $(151,5)$ debido al alto número de citas de los artículos que destacan en cada etapa (nueve artículos con 1.364 citas). 
Tabla 12

Referencias más citadas de mobile learning en la etapa de Educación Primaria

\begin{tabular}{|c|c|c|c|c|}
\hline Autores & Año & Título & Medio de publicación & Citas \\
\hline $\begin{array}{l}\text { Furió, D., González- } \\
\text { Gancedo, S., Juan, M.C., } \\
\text { Seguí, I. y Rando, N }\end{array}$ & 2013 & $\begin{array}{l}\text { Evaluation of learning outcomes } \\
\text { using an educational iPhone } \\
\text { game vs. traditional game }\end{array}$ & $\begin{array}{l}\text { Computers and Education, } \\
64, \text { pp. } 1-23\end{array}$ & 54 \\
\hline $\begin{array}{l}\text { Guerrero, L.A., Ochoa, S. } \\
\text { y Collazos, C }\end{array}$ & 2010 & $\begin{array}{l}\text { A mobile learning tool for } \\
\text { improving grammar skills }\end{array}$ & $\begin{array}{l}\text { Procedia - Social and } \\
\text { Behavioral Sciences } \\
2(2) \text {, pp. } 1735-1739\end{array}$ & 16 \\
\hline $\begin{array}{l}\text { Sánchez-Prieto, J.C., } \\
\text { Migueláñez, S.O. y } \\
\text { García-Peñalvo, F.J }\end{array}$ & 2014 & $\begin{array}{l}\text { ICTs integration in education: } \\
\text { Mobile learning and the } \\
\text { technology acceptance model } \\
\text { (TAM) }\end{array}$ & $\begin{array}{l}\text { ACM International } \\
\text { Conference Proceeding } \\
\text { Series } \\
\text { pp. } 683-687\end{array}$ & 10 \\
\hline
\end{tabular}

Tabla 13

Referencias más citadas de mobile learning en la etapa de Educación Secundaria

\begin{tabular}{|c|c|c|c|c|}
\hline Autores & Año & Título & Medio de publicación & Citas \\
\hline $\begin{array}{l}\text { McKinney, D., Dyck, JL. } \\
\text { y Luber, ES }\end{array}$ & 2009 & $\begin{array}{l}\text { iTunes University and the } \\
\text { classroom: Can podcasts replace } \\
\text { Professors? }\end{array}$ & $\begin{array}{l}\text { Computers and Education } \\
52(3) \text {, pp. } 617-623\end{array}$ & 134 \\
\hline $\begin{array}{l}\text { Vavoula, G., Sharples, } \\
\text { M., Rudman, P., Meek, J. } \\
\text { y Lonsdale, P }\end{array}$ & 2009 & $\begin{array}{l}\text { Myartspace: Design and evaluation } \\
\text { of support for learning with } \\
\text { multimedia phones between } \\
\text { classrooms and museums }\end{array}$ & $\begin{array}{l}\text { Computers and Education } \\
53(2) \text {, pp. } 286-299\end{array}$ & 113 \\
\hline $\begin{array}{l}\text { Hsu, C.K., Hwang, G.J. y } \\
\text { Chang, C.K }\end{array}$ & 2013 & $\begin{array}{l}\text { A personalized recommendation- } \\
\text { based mobile learning approach to } \\
\text { improving the reading performance } \\
\text { of EFL students }\end{array}$ & $\begin{array}{l}\text { Computers and Education } \\
63 \text {, pp. } 327-336\end{array}$ & 69 \\
\hline $\begin{array}{l}\text { Gedik, N., Hanci- } \\
\text { Karademirci, A., Kursun, } \\
\text { E. y Cagiltay, K. }\end{array}$ & 2012 & $\begin{array}{l}\text { Key instructional design issues in a } \\
\text { cellular phone-based mobile } \\
\text { learning project }\end{array}$ & $\begin{array}{l}\text { Computers and Education } \\
58(4) \text {, pp. } 1149-1159\end{array}$ & 38 \\
\hline Yen, J.C. y Lee, C.Y & 2011 & $\begin{array}{l}\text { Exploring problem solving patterns } \\
\text { and their impact on learning } \\
\text { achievement in a blended learning } \\
\text { environment }\end{array}$ & $\begin{array}{l}\text { Computers and Education } \\
56(1) \text {, pp. } 138-145\end{array}$ & 32 \\
\hline
\end{tabular}

Tabla 14

Referencias más citadas de mobile learning en la etapa de Educación Superior

\begin{tabular}{|c|c|c|c|c|}
\hline Autores & Año & Título & Medio de publicación & Citas \\
\hline Motiwalla, L.F & 2007 & $\begin{array}{l}\text { Mobile learning: A framework and } \\
\text { evaluation }\end{array}$ & $\begin{array}{l}\text { Computers and Education } \\
49(3) \text {, pp. } 581-596\end{array}$ & 408 \\
\hline Evans, C. & 2008 & $\begin{array}{l}\text { The effectiveness of m-learning in } \\
\text { the form of podcast revision } \\
\text { lectures in higher education }\end{array}$ & $\begin{array}{l}\text { Computers and Education } \\
50(2) \text {, pp. 491-498 }\end{array}$ & 329 \\
\hline Gikas, J. y Grant, M.M & 2013 & $\begin{array}{l}\text { Mobile computing devices in higher } \\
\text { education: Student perspectives on } \\
\text { learning with cellphones, } \\
\text { smartphones \& social media }\end{array}$ & $\begin{array}{l}\text { Internet and Higher } \\
\text { Education } \\
\text { 19, pp. 18-26 }\end{array}$ & 228 \\
\hline $\begin{array}{l}\text { Cheon, J., Lee, S., } \\
\text { Crooks, S.M. y Song, J }\end{array}$ & 2012 & $\begin{array}{l}\text { An investigation of mobile learning } \\
\text { readiness in higher education } \\
\text { based on the theory of planned } \\
\text { behavior }\end{array}$ & $\begin{array}{l}\text { Computers and Education } \\
59(3) \text {, pp. 1054-1064 }\end{array}$ & 187 \\
\hline $\begin{array}{l}\text { El-Hussein, M.O.M. y } \\
\text { Cronje, J.C }\end{array}$ & 2010 & $\begin{array}{l}\text { Defining mobile learning in the } \\
\text { higher education landscape }\end{array}$ & $\begin{array}{l}\text { Educational Technology and } \\
\text { Society } \\
13(3), \text { pp. } 12-21\end{array}$ & 145 \\
\hline
\end{tabular}




\section{Discusión y conclusiones}

En función de las aportaciones de estudios bibliográficos previos en la base de datos Scopus (RodríguezGarcía y Martínez, 2017; Mengual, Vázquez y López, 2017), se ha atendido a las nueve variables recogidas por estos autores para la revisión bibliométrica de la literatura científica. Se constata que la mayoría de las publicaciones sobre mobile learning corresponden a la etapa de Educación Superior, lo cual es síntoma de que en la Universidad es donde primero ocurren los cambios metodológicos, debido a la doble vertiente del profesorado (docente e investigador) y la flexibilidad de acceso a la muestra. En el lado contrario se encuentra la etapa de Educación Infantil donde la producción es escasa, lo que denota a priori una falta de interés por la implementación de esta metodología en dicha etapa. Esto puede deberse a que los niños más pequeños (0-6 años), no tienen dispositivos móviles o no se les permite tenerlo. Además, el tiempo adecuado de uso del dispositivo móvil en la población de niños entre 2-5 años es de una hora (American Academy of Pediatrics, 2016).

Por otro lado, el área de CC. Computacionales es la que acapara más producción en todas las etapas educativas, seguido de las CC. Sociales donde las Ciencias de la Educación tiene su cabida y deberían encabezar la producción científica sobre la implementación de esta metodología. No obstante, la literatura relacionada con las $\mathrm{CC}$. Sociales se acerca o distancia de las CC. Computacionales en función de la etapa: CC. Sociales 20\% en Educación Infantil (CC. Computacionales: $60 \%$ ), CC. Sociales 33\% en Educación Primaria (CC. Computacionales: $46 \%$ ), CC. Sociales $41 \%$ en Educación Secundaria (CC. Computacionales: $44 \%$ ) y CC. Sociales 39\% en Educación Superior (CC. Computacionales: $43 \%$ ). En consideración, con el creciente interés por la investigación del mobile learning desde el ámbito de las Ciencias de la Educación (englobadas dentro de las CC. Sociales) posiblemente en los próximos años supere la producción derivada de las CC. Computacionales. En cambio, el ámbito de la Medicina no se ha visto reflejado en este estudio, por lo que a priori no existe investigaciones o experiencias publicadas sobre el uso de una metodología basada en el uso de los dispositivos móviles.

Centrando la atención en los años de publicación, la mayor parte de las publicaciones se concentran entre los años 2015-2017 en Educación Infantil, Educación Primaria y Educación Superior. En las tres primeras etapas (Infantil, Primaria y Secundaria) la producción científica se sitúa en la etapa de precursores que destacaba Price (1986), encontrándose en una fase inicial de la literatura que todavía no ha obtenido su crecimiento exponencial y consolidación. De modo que no es de extrañar que el mobile learning sea un tema candente y aumente la producción acerca de esta metodología en los años venideros. La única etapa en la que ha empezado a consolidarse el mobile learning es Educación Superior donde ha tenido un mayor desarrollo y se sitúa en una fase de crecimiento lineal.

En este sentido, las aportaciones a jornadas y congresos son los primeros indicadores de las innovaciones metodológicas que empiezan a emerger, resaltándose en este estudio bibliométrico que la producción científica más numerosa proviene de las aportaciones a conferencias en las etapas de Educación Primaria y Educación Superior, mientras que los artículos predominan en Educación Infantil y Educación Secundaria. De estos datos podría deducirse que en primera instancia el mobile learning está teniendo una aceptación previa en los eventos científicos, a la vez que se materializa en la producción de artículos. De modo que las aportaciones a congresos son un indicador de aumento de la producción en los próximos años. Así pues, se prevé una mayor producción de artículos en la etapa de Educación Superior como resultado de la consolidación de la metodología. Mientras que en Educación Infantil, Educación Primaria y Educación Secundaria, al encontrarse en una fase inicial de la literatura científica aumentará en ambas direcciones.

En relación a la procedencia de la literatura científica destaca la conferencia $A C M$ International Conference Proceeding Series que se sitúa entre las que más referencias obtienen en Educación Primaria con tres documentos y en Educación Secundaria con otros tres documentos. Como revista resalta Computers and Education con 12 documentos en Educación Secundaria y otros 12 en Educación Superior. Además, es la que mayor índice de impacto presenta (40 puntos en Educación Secundaria y 99,91 puntos en Educación Superior). En consideración, se constata el interés de revistas como Computers and Education en la metodología mobile learning sitúandose como la revista con mayor impacto y de referencia para todos aquellos interesados en esta temática.

En cuanto a las palabras clave relacionadas con los tópicos de búsqueda, resalta el descriptor "Education" en las etapas de Educación Infantil, Educación Primaria y Educación Superior, hecho que contrasta con la prevalencia del área de CC. Computacionales. Podría dilucidarse que esta área presenta interés por el ámbito educativo y asimismo, el cómputo total de distintos ámbitos de conocimiento utilizan este descriptor como principal en sus contribuciones. 
Poniendo el foco de atención en las instituciones de referencia, excepto en Educación Infantil destacan en todas las etapas las universidades españolas (Universidad de Salamanca, Universidad Politécnica de Cataluña y Universidad Pompeu Fabra), situándose entre las cinco primeras instituciones en la literatura sobre mobile learning en Educación Primaria, Educación Secundaria y Educación Superior. De igual forma, España se encuentra entre los cinco primeros países en la producción científica en estas etapas educativas. Sin embargo, se generan divisiones en las etapas educativas, donde predominan ciertos países en función de cada una. En concreto, en Educación Infantil y Educación Primaria destaca Grecia, mostrando los investigadores griegos cierto interés por las etapas educativas iniciales. En Educación Secundaria, España se sitúa a la cabeza y aparecen países como Taiwán, Austria, Reino Unido y Estados Unidos. Taiwán y Austria ponen su atención principalmente en esta etapa educativa, puesto que no aparecen en puestos relevantes en la publicación sobre mobile learning en las otras etapas educativas. Por último, en Educación Superior Reino Unido lidera las aportaciones, seguido de Estados Unidos, Australia, España y Malasia. Con base en las otras etapas educativas, aparece por primera vez Australia y Malasia, donde sus trabajos sobre mobile learning se han centrado principalmente en la Educación Superior.

A pesar del número de documentos que obtienen los países, siendo los mayores productores Grecia en Educación Infantil, España en Educación Primaria y Educación Secundaria y Reino Unido en Educación Superior, el mayor índice de impacto en Educación Secundaria y Educación Superior lo obtiene Estados Unidos. A este respecto, la literatura científica española sobre mobile learning se encuentra en unos niveles óptimos en calidad y cantidad, mientras que países como Reino Unido, Taiwán, Australia y Estados Unidos tienen mayor índice de impacto y citas. Este interés de los investigadores españoles sobre el mobile learning, se justifica principalmente en el desarrollo de las TIC estos últimos años y los diversos programas españoles que incentivan la introducción de las TIC en el aula, como el Plan Escuela TIC 2.0 de Andalucía y a nivel estatal el programa Escuela 2.0 promovido por el Ministerio de Educación, Cultura y Deporte (Consejería de Educación de la Junta de Andalucía, 2011; Ministerio de Educación, Cultura y Deporte, 2009).

Respecto a los autores con más producción, destacan distintas personas en cada etapa, cosechando el que más 11 títulos sobre la temática mobile learning en Educación Superior (Farley, H y Murphy, A). Además, aquellos autores que coinciden en el número de documentos y citas suelen firmar juntos los trabajos. Algunos de los que presentan mayor número de referencias coinciden entre las referencias con más citación. En Educación Primaria, los tres autores trabajan juntos en la producción científica recogida, resaltando entre las referencias con más citas "ICTs integration in education: Mobile learning and the technology acceptance model (TAM)". Y en Educación Secundaria, el autor Hwang, G.J coincide con uno de los artículos más citados (69 citas). Por su parte, destaca como la revista que más aparece entre las publicaciones de impacto Computers and Education, lo cual concuerda como hacíamos mención en el apartado metodológico, con algunos artículos (O'Bannon y Thomas, 2014; Zydney y Warner, 2015; Han y Shin, 2016) que recogen el descriptor "Mobile Learning".

Finalmente, los datos confirman el auge de la metodología mobile learning en todas las etapas educativas, resaltando la etapa de Educación Superior, donde se está consolidando el cuerpo teórico. Aunque los informes Horizon apunten a la implementación del mobile learning en menos de un año (Alexander et al., 2019), consideramos que todavía queda camino por recorrer en la investigación enfocada a las etapas educativas iniciales y medias. Así pues, la aplicación y consolidación de los dispositivos móviles se establece que ocurrirá primero en la Educación Superior y posteriormente irá implementándose en niveles inferiores conforme avance la producción científica en estas etapas a nivel mundial.

En definitiva, el estudio bibliométrico sobre la producción científica en Scopus proporciona unos datos de interés para la comunidad científica. Por un lado, en cuanto al conocimiento que ofrecen los datos acerca del fenómeno mundial del mobile learning y por otro lado, en relación a la tendencia de consolidación del mobile learning en cada etapa educativa.

\section{Apoyos}

Ministerio de Educación, Cultura y Deporte (FPU16/01762)

\section{Referencias}

Adams, S., Brown, M., Dahlstrom, E., Davis, A., DePaul, K., Díaz, V. y Pomerantz, J. (2018). NMC Horizon Report: 2018 Higher Education Edition. Louisville, CO: EDUCAUSE.

Adams, S., Cummins, M., Davis, A., Freeman, A., Hall, C. y Ananthanarayanan, V. (2017). NMC Horizon Report: 2017 Higher Education Edition. Austin, Texas: The New Media Consortium. 
Alexander, B., Ashford-Rowe, K., Barajas-Murphy, N., Dobbin, G., Knott, J., McCormack, M., ... Weber, N. (2019). EDUCAUSE Horizon Report: 2019 Higher Education Edition. Louisville, CO: EDUCAUSE.

American Academy of Pediatrics (2016). American Academy of Pediatrics Announces New Recommendations for Children's Media Use. Retrieved from https://goo.gl/KAxkhH

Aznar, I., Cáceres, M.P. y Romero, J.M. (2018). Indicadores de calidad para evaluar buenas prácticas docentes de «mobile learning» en Educación Superior. Education in the Knowledge Society (EKS), 19(3), 53-68. https://doi.org/10.14201/eks20181935368

Brazuelo, F. y Gallego D.J. (2014). Estado del Mobile Learning en España. Educar em Revista, 4, 99-128. HTTPS://doi.org/10.1590/0104-4060.38646

Consejería de Educación de la Junta de Andalucía (2011). Escuela TIC 2.0. Disponible en

https://www.juntadeandalucia.es/export/drupaljda/PlanEscuela.pdf Corlett, D., Sharples, M., Bull, S. y Chan, T. (2005). Evaluation of a mobile learning organiser for university students. Journal of Computer Assisted learning, 21, $162-170$. https://doi.org/10.1111/j.1365-2729.2005.00124.x

Glanzel, W. y Schoepflin, U. (1999). A bibliometric study of reference literature in the sciences and social sciences. Information processing \& management, 35(1), 31-44.

https://doi.org/10.1016/s0306-4573(98)00028-4

González, R. y Gutiérrez, A. (2017). Competencias Mediática y Digital del profesorado e integración curricular de las tecnologías digitales. Revista Fuentes, 19(2), 57-67. https://doi.org/10.12795/revistafuentes.2016.19.2.04

Han, I. y Shin, W.S. (2016). The use of a mobile learning management system and academic achievement of online students. Computers \& Education, 102, 79-89.

https://doi.org/10.1016/j.compedu.2016.07.003

INTEF (2017). Resumen informe Horizon: edición 2017 educación superior. Madrid: Ministerio de Educación, Cultura y Deporte.

Jiménez, D., Mora, M. y Cuadros, R. (2016). La importancia de las nuevas tecnologías en el proceso educativo.

Propuesta didáctica TIC para ELE: mELEndien7dias. Revista Fuentes, 18(2), 209-233.

https://doi.org/10.12795/revistafuentes.2016.18.2.07

Johnson, L., Adams, S., \& Cummins, M. (2012). The NMC Horizon Report: 2012 Higher Education Edition. The New

Media Consortium.

Johnson, L., Adams, S., Cummins, M., Estrada, V., Freeman, A., \& Hall, C. (2016). NMC Horizon Report: 2016 Higher Education Edition. The New Media Consortium.

Johnson, L., Adams, S., Cummins, M., Estrada, V., Freeman, A. \& Ludgate, H. (2013). NMC Horizon Report: 2013 Higher Education Edition. The New Media Consortium.

Johnson, L., Adams, S., Estrada, V., \& Freeman, A. (2014). NMC Horizon Report: 2014 K-12 Edition. The New Media Consortium.

Johnson, L., Adams, S., Estrada, V., \& Freeman, A. (2015). NMC Horizon Report: 2015 Higher Education Edition. The New Media Consortium.

López, E., Vázquez, E., \& Román, P. (2015). Análisis e implicaciones del impacto del movimiento MOOC en la comunidad científica: JCR y Scopus (2010-13). Comunicar, 22(44), 73-80. https://doi.org/10.3916/C44-2015-08.

Mengual, S., Vázquez, E., \& López, E. (2017). La productividad científica sobre MOOC: aproximación bibliométrica 2012-2016 a través de SCOPUS. RIED. Revista Iberoamericana de Educación a Distancia, 20(1), 39-58.

https://doi.org/10.5944/ried.20.1.16662

Ministerio de Educación, Cultura y Deporte (2009). Programa Escuela 2.0. Disponible en

https://www.lamoncloa.gob.es/Paginas/archivo/040409-enlace20.aspx

O'Bannon, B.W., \& Thomas, K. (2014). Teacher perceptions of using mobile phones in the classroom: Age matters. Computers \& Education, 74, 15-25.

https://doi.org/10.1016/j.compedu.2014.01.006.

Price, D.J.S. (1986). Little Science, big science... and beyond. Columbia University Press.

Ramos, A.I., Herrera, J.A., \& Ramírez, M.S. (2010). Desarrollo de habilidades cognitivas con aprendizaje móvil: un estudio de casos. Comunicar, 17(34), 201-209.

https://doi.org/10.3916/c34-2010-03-20.

Rodríguez-García, A.M., \& Martínez, N. (2017). La competencia digital en la base de Scopus: un estudio de metaanálisis. En J. Silva (Ed.), EDUcación y TECnología: una mirada desde la Investigación e Innovación. Actas del XX Congreso Internacional EDUTEC 2017 (pp. 676-678). Centro de Innovación e Investigación en Educación y Tecnología (CIIET) de la Universidad de Santiago de Chile.

Romero, J.M., \& Rodríguez-García, A.M. (2017). Digitalización de recursos patrimoniales mediante códigos QR: los dispositivos digitales móviles para la difusión cultural y artística en educación. En L. Bocanegra y A. García (Eds.), Con la Red / En la Red. Creación, Investigación y Comunicación Cultural y Artística en la era Internet (pp. 595-612).

Universidad de Granada 
Romero, J.M., Trujillo, J.M., \& Rodríguez-García, A.M. (2018). Bring Your Own Device to The Classroom: Uses Of The Mobile Phone For The University Training. TOJED. The Turkish Online Journal of Educational Technology, 1(special issue for INTE-ITICAM-IDEC), 254-260.

Sharples, M., Corlett, D., \& Westmancott, O. (2002). The Design and Implementation of a Mobile Learning Resource. Personal and Ubiquitous Computing, 6, 220-234. https://doi.org/10.1007/s007790200021

Urrútia, G., \& Bonfill, X. (2010). Declaración PRISMA: una propuesta para mejorar la publicación de revisiones sistemáticas y metaanálisis. Medicina Clínica, 135(11), 507-511.

https://doi.org/10.1016/j.medcli.2010.01.015

Zydney, J.M. y Warner, Z. (2015). Mobile apps for science learning: Review of research. Computers \& Education, 94,1 17.

https://doi.org/10.1016/j.compedu.2015.11.001 